\title{
Análisis de la demanda de los visitantes que realizaron turismo idiomático en la ciudad de Cuenca-Ecuador en el año 2015
}

\section{Analysis of the visitors' demand who searched the idiomatic tourism in the city of Cuenca-Ecuador in 2015}

\author{
Cristina Eulalia Campoverde Urgilés ${ }^{1}$, María Inés Landi Pauta ${ }^{2}$.
}

凶 Autorde correspondencia: ccampoverdemtu@es.uazuay.edu.ec,infer_28@hotmail.com

1. Universidad del Azuay. Rafael Zeas y Miguel López. Cuenca-Ecuador.

2. Escuela Panamá. Paccha-Ecuador.

Artículo original de la ponencia presentada en el IV CONGRETUR - "El impacto del turismo en el desarrollo del Ecuador", Universidad Central del Ecuador, 21 al 24 de noviembre de 2018

\begin{abstract}
Resumen
En los últimos años en la ciudad de Cuenca-Ecuador se ha incrementado la demanda de extranjeros que vienen a la ciudad a realizar estudios del idioma español. Estos extranjeros son conocidos como turistas idiomáticos, tienen características específicas que los diferencian de los turistas generales, por su tiempo de estancia, alojamiento, interés en la cultura del sitio a estudiar, atractivos complementarios. Por la creciente demanda de turistas idiomáticos en la ciudad se determinó el perfil de los mismos, realizando un análisis socio-demográfico, motivacional, de preferencias, tendencias y estacionalidad, a través de encuestas, las mismas que se realizaron en los centros de enseñanza del idioma español de la ciudad de Cuenca: Amauta Spanish School, Estudio Sampere, CEDEI (Centro de Estudios Iberoamericanos), Simón Bolivar Spanish School, Yanapuma Spanish School y en el programa de español para extranjeros que maneja el Instituto de Lengua de la Universidad de Cuenca. Se trabajó con 299 encuestas, en las que se determinó el tipo de alojamiento, la estacionalidad, los atractivos de la ciudad, el costo involucrado en llevar a cabo la actividad y la forma específica en la que conocieron el destino de Cuenca.
\end{abstract}

Palabras clave: Perfil, Turismo idiomático, Cuenca, Estancia.

\begin{abstract}
In recent years, in the city of Cuenca-Ecuador, the demand of foreigners who come to the city to conduct studies of the Spanish language has increased. These foreigners are known as idiomatic tourists. They have specific characteristics that differentiate them from the other tourists for their time to stay, accommodation, interest in the culture of the site to be studied, complementary attractions. Due to the growing demand for idiomatic tourists in the city, the profile of these people was determined by a socio-demographic, motivational, preferences, trends and stationed analysis through surveys, which were carried out in the educational centers of the city of Cuenca: Amauta Spanish School, Estudio Sampere, CEDEI (Center for Ibero-American Studies), Simón Bolivar Spanish School, Yanapuma Spanish School and in the Spanish program for foreigners managed by the Language Institute of the Universidad de Cuenca. We applied 299 surveys and the type of accommodation, the stationer, the attractions of the city, the cost involved in carrying out the activity, and the specific form in which the tourists researched about Cuenca destination were determined.
\end{abstract}

Keywords: Profile; Idiomatic tourist; Cuenca; Stay. 


\section{Introducción}

Para conocer la relación del turismo idiomático con otras tipologías de turismo (Figura 1), se hace referencia al cuadro que Pawlowska (2011, p.
12) utiliza en su investigación "El turismo académico: un análisis económico para el caso Galicia".

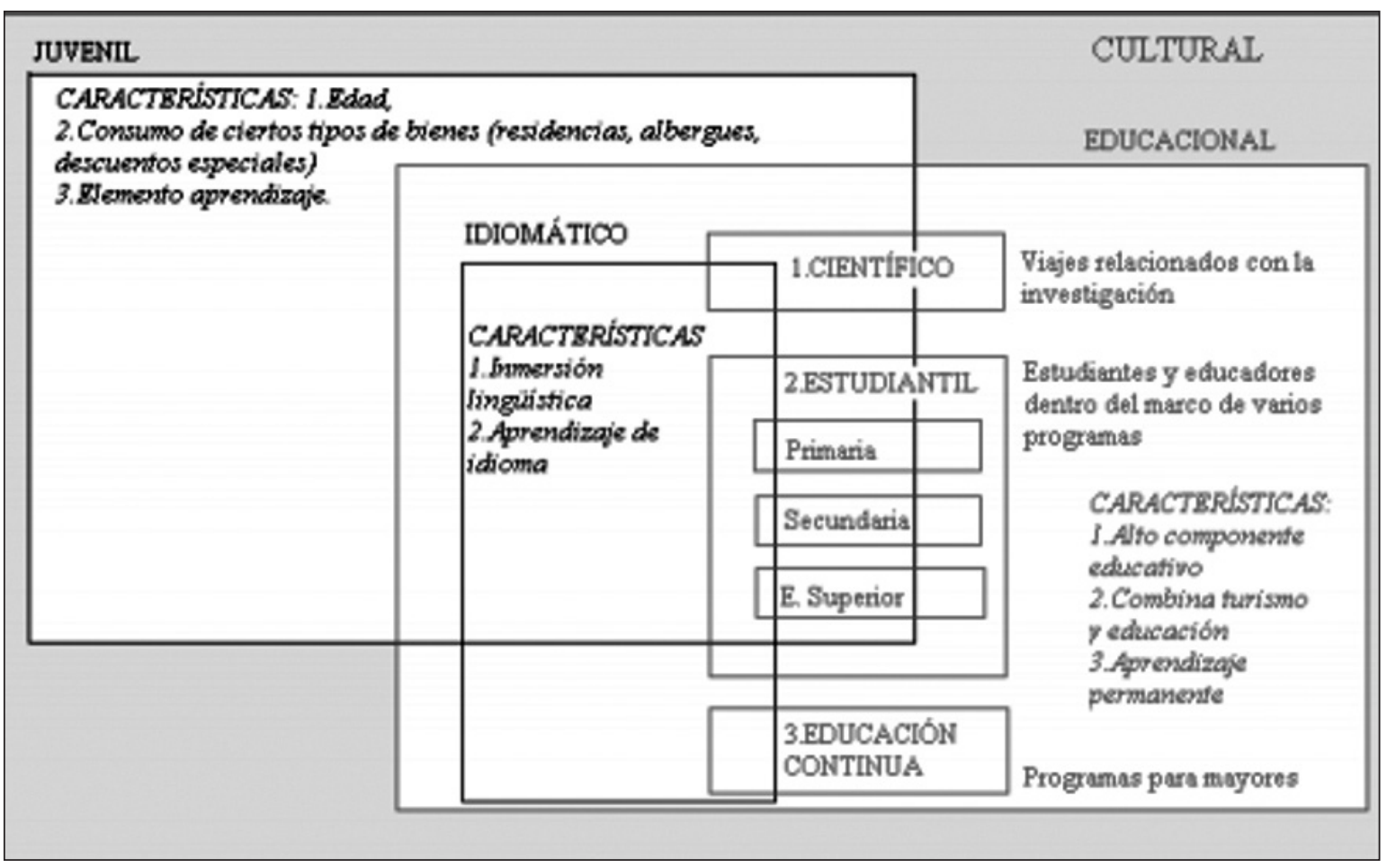

Figura 1. Relación de las tipologías del turismo.

Luego de apreciar el cuadro podemos ver que Pawlowska divide al turismo cultural, que es "definido como aquel viaje turístico motivado por conocer, comprender y disfrutar el conjunto de rasgos y elementos distintivos, espirituales y materiales, intelectuales y afectivos que caracterizan a una sociedad... de un destino específico" (Secretaría de Turismo, 2015), en dos grandes grupos: turismo académico y turismo juvenil y en la fusión de los dos se encuentra el turismo idiomático.

Pawlowska (2011, p. 12) menciona que la Confederación Mundial de Viajes Educacionales para Jóvenes y Estudiantes y la Organización Mundial del Turismo hacen una aproximación conceptual sobre lo que se concibe como turismo juvenil y menciona que "es un nicho de mercado relacionado con unos determinados productos o estilos de viaje, tales como turismo independiente, 'turismo mochilero', turismo de aventura, turismo de idiomas o prácticas profesionales... En cambio, Montaner (1991, p. 59) considera al turismo juvenil como "parte del turismo social y además subdivide a esta tipología en turismo escolar, juvenil y universitario".

Por otro lado, Pawlowska (2011, p. 20) utiliza la afirmación de Montull I Aced, quien dice que el turismo académico puede referirse a "Todas las estancias de duración inferior a un año, realizadas en los centros de enseñanza superior fuera del país de residencia habitual y cuyo objetivo principal... sería la realización de cursos relacionados con una titulación universitaria y/o la asistencia a cursos de idiomas.

Luego de precisar conceptos sobre la subdivisión que menciona Palowska (2011), es necesario enfocarse en el turismo idiomático, debido a que esta tipología de turismo es considerado una de las nuevas formas de turismo según Moral Cuadra y Orgaz (2013) en su estudio "Las nuevas tipologías del turismo en España: el caso de Andalucía" (Cuadra \& Orgaz, 2013); y la OMT lo define como "Las actividades que realizan las personas durante sus viajes y estancias en lugares distintos al de su entorno natural por un periodo de tiempo consecutivo inferior a un año, con el fin de hacer la inmersión lingüísti- 
ca en un idioma distinto al de su entorno natural" (Soto, 2012, p. 58). Este concepto fue tomado de la investigación de Soto (2012), sobre "La movilidad estudiantil internacional como turismo académico".

Es necesario también citar la definición que utiliza la Universidad "Antonio Nebrija" en sus Primeras Jornadas Internacionales de Lenguas Aplicadas al Turismo, en donde no se habla de un turismo idiomático como tal, sino se utiliza el término turismo lingüístico, el cual se entiende como "una modalidad de viajes de estudio y ocio, que buscan cada vez más personas, para aprovechar las vacaciones aprendiendo o mejorando su competencia en una lengua".

Si se habla de esta tipología de turismo en España, es necesario mencionar dos subdivisiones, las cuales dependen del objetivo de su viaje:

- ERASMUS: Son estudiantes que van a España a proseguir estudios universitarios por convenios universitarios por periodos de 3 a 6 meses.

- NO ERASMUS: aquellos que tienen como objetivo principal el aprender el idioma. Generalmente lo hacen a lo largo del año, durante sus períodos vacacionales y su estancia dura un tiempo aproximado de 3 meses.

Además, se cita a Baraló (2007), quien dice que en el turismo idiomático se incluyen "los viajes que se realizan al extranjero para poner en práctica las actividades relacionadas con el aprendizaje de una lengua... la cual tiene cada vez más demanda... y lo considera un fenómeno asociado sobre todo con el período de vacaciones...". Goeldner \& Ritchie (2011) consideran que el interés de conocer el idioma extranjero es una fuerza de motivación en el turismo y que esta afirmación es completamente verdadera en el caso del estudiante que viaja a un país para practicar un idioma y para familiarizarse con su uso coloquial.

Dentro del turismo idiomático también se encuentra una nueva propuesta conocida como "turilingüismo"; que es una tipología que abarca los tres factores más importantes del fenómeno: el lingüístico, el turístico y el económico. Por ello se puede definir al "turilingüismo" como "el campo que desarrolla la relación entre el estudio de una lengua y su potencial económico por su capacidad para atraer al turismo" (Ganfornina, 2005, p. 4).

Se conoce que en países como Reino Unido, Francia y Alemania se inició el turismo idiomático como una actividad turística que está incluida y promocionada dentro del turismo cultural (Moral Cuadra \& Orgaz, 2013, p. 17). Además, en la investiga- ción realizada por Meza (2013) sobre "Análisis del club del producto de turismo idiomático en la ciudad de Málaga", se indica que, los primeros datos que se tienen sobre turismo idiomático son provenientes de instituciones europeas, tales como British Council en Gran Bretaña, Alianza Francesa en Francia y el Instituto Goethe en Alemania. Así también se considera de gran importancia el Instituto Cervantes en España y el programa Erasmus.

En la ciudad de Cuenca-Ecuador este tipo de turismo ha tenido una demanda creciente en los centros de enseñanza, pero no se tenía definido un perfil. Es por eso que el presente artículo pretende entregar los resultados obtenidos en la investigación "El análisis de la demanda de los visitantes que realizaron turismo idiomático en la ciudad de Cuenca en el año 2015", que fue el año en que se desarrolló la investigación.

\section{Materiales y métodos}

Para determinar el perfil de los turistas idiomáticos que visitaron la ciudad de Cuenca-Ecuador se aplicó la metodología cuantitativa, se efectuaron encuestas a los estudiantes de los centros de enseñanza Amauta Spanish School, Estudio Sampere, Simón Bolivar Spanish School, CEDEI (Centro de estudios Iberoamericanos), Yanapuma Spanish School y del Programa de español para extranjeros que maneja el Instituto Universitario de Lengua y Literatura de la Universidad de Cuenca. Las encuestas fueron el principal recurso utilizado, debido a que fue necesario recopilar información de los extranjeros que aprendieron el idioma español en los centros de enseñanza de la ciudad de Cuenca, por la gran cantidad de interrogantes que se tenían en cuanto a los motivos y preferencias que causaban la llegada de extranjeros, las encuestas fueron un método más efectivo y eficaz para conseguir el objetivo.

Para validar la encuesta, primero se realizó a un grupo focal que sirvió para verificar si los estudiantes extranjeros, entendían con claridad las preguntas formuladas y también para calcular el tiempo aproximado que los encuestados necesitarían para llenar la misma. El grupo focal fue muy provechoso ya que finalmente se realizaron los respectivos cambios a las encuestas para un mejor entendimiento y aplicación.

Las encuestas fueron realizadas en dos idiomas: español e inglés y contenían 22 preguntas, las mismas que estaban divididas en 3 secciones: 10 preguntas correspondientes a información socio demográfica, que englobaba: género, edad, país de origen, el tiempo de permanencia, presupuesto para comida, aloja- 
miento, entretenimiento y viajes, y sus conocimientos de idiomas; además, 8 preguntas correspondientes a motivaciones y preferencias, sobre medios de información para realizar turismo idiomático en la ciudad de Cuenca, experiencias de turismo y viajes a las distintas regiones del Ecuador, formas de viajar, lugares de hospedaje; y, sus percepciones acerca del servicio ofrecido en los centros de enseñanza del idioma español y por la Universidad de Cuenca, la ciudad y el país. Finalmente, 4 preguntas abiertas para conocer preferencias, sugerencias o recomendaciones de los estudiantes para con los centros de enseñanza de idiomas de la ciudad en general. El tiempo estimado para llenar la encuesta fue de 10 a 15 minutos, lo cual dependía del idioma de elección.

Dentro de las preguntas mencionadas en los párrafos anteriores, constaban 4 preguntas que utilizaron la escala de Likert, dicha escala mide actitudes o predisposiciones individuales en contextos sociales, para la interpretación de esta escala se realizó una sumatoria con las respuestas obtenidas de cada parámetro, el encuestado debía escoger parámetros de 1 a 5, donde 1 era igual a Para nada y 5 era igual a Mucho. Para la tabulación de las encuestas se utilizó el programa SPPS 19, que permite obtener datos estadísticos de una base de datos, para la interpretación y consolidación de resultados de la investigación, además se utilizó Excel para obtener mejores graficas de los datos obtenidos.

La mayoría de encuestas fueron aplicadas en los meses de febrero, marzo, mayo y junio del año 2015, de acuerdo a la afluencia de estudiantes en los centros de enseñanza, el mes de abril fue un mes considerado como temporada baja, debido a que el número de estudiantes en cada centro de enseñanza no superaba los 5 alumnos aproximadamente e incluso en algunos casos los estudiantes se mantenían desde meses anteriores en los centros. Es importante mencionar que se tuvo que esperar por los grupos de estudiantes que llegaron a los diferentes centros de enseñanza del idioma, ya que algunos de ellos tenían convenios con centros de estudio internacionales o universidades, las cuales enviaban a los estudiantes en diferentes temporadas y diverso número.

Al ser las encuestas el principal método de análisis a utilizar en la investigación, primero se determinó la muestra de encuestas que se debían aplicar para conseguir el objetivo general de esta investigación que era: Determinar el perfil de los visitantes que escogen a Cuenca-Ecuador como un destino de turismo idiomático, pero para ello fue preciso obtener la población total de los estudiantes que aprendieron español en las escuelas anteriormente citadas durante el año 2013. Se tomó como base de estudio el año 2013, debido a que la propuesta de analizar la demanda de los visitantes que realizaron turismo idiomático en la ciudad de Cuenca-Ecuador, se realizó en el año 2014; además, el momento en que el tema de investigación fue aprobado, los centros de enseñanza del idioma español, aún no tenían tabulados los datos estadísticos de sus estudiantes del año 2014.

Se procedió a definir el número de la población de estudiantes del año 2013 que tuvieron los centros de enseñanza, obteniendo un total de 1.405 extranjeros, este dato fue obtenido directamente de las escuelas de español que se investigaron mediante entrevista a sus directores. Para definir la muestra estadística se procedió a aplicar la siguiente formula estadística:

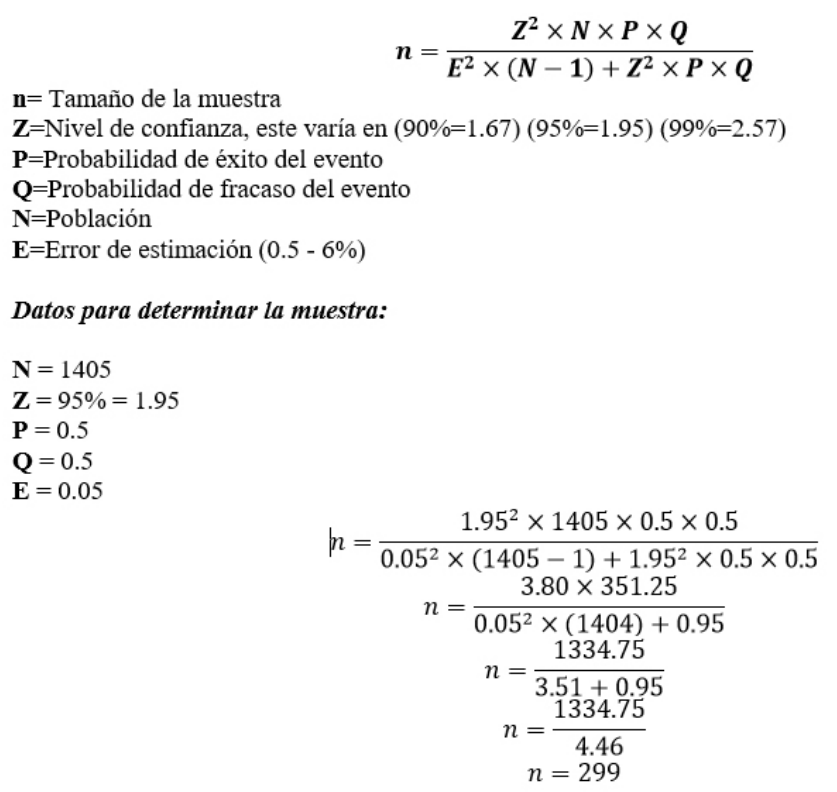

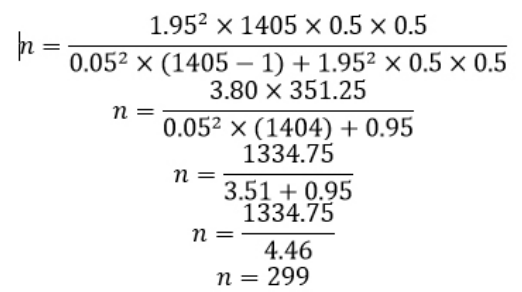


Luego de aplicar la fórmula para conocer la muestra se obtuvo un total de 299 encuestas que se realizaron con las cuales se tuvo un $95 \%$ de confiabilidad.

Tomando en cuenta la amplitud de la investigación y la necesidad de recurrir a información existente, también se utilizó, metodología cualitativa, en la cual se aplicó el método de estudio de casos, porque existieron documentos útiles para la realización de este trabajo, los mismos que han sido desarrollados en países latinos, aportando significativamente para conseguir el objetivo de la investigación. Dentro de los artículos y libros utilizados se puede mencionar los siguientes: "Análisis de club de producto de turismo idiomático en la ciudad de Málaga" realizado por Meza (2011); también, "El turismo idiomático en España como potenciador cultural en ciudades patrimoniales: el caso de Alcalá de Henares", realizado por Pardo (2011), "El turismo académico, un análisis económico para el caso de Galicia", realizado por Pawlowska (2011), "El turismo idiomático en la Argentina. Principales rasgos de la demanda", documento realizado por Leighton, Arcuri \& Jimeno (2012), "El turismo idiomático como industria cultural" de Leighton, Adriani, \& Schüler (2011), también se menciona a Cohen \& Cooper (1986), con su investigación titulada "Language and tourism", también se utilizó la investigación de Baraló (2015) "El valor del idioma como recurso turístico, económico y cultural "; así mismo el artículo de Alonso (2006) sobre la "Naturaleza económica de la lengua". Y se analizó otro artículo realizado por Adriani et al. (2014) sobre "Una aproximación del turismo idiomático en Argentina".

\section{Resultados}

Luego de aplicar y analizar las encuestas se determinó que los turistas idiomáticos que visitaron la ciudad de Cuenca-Ecuador en el año 2015 fueron en su mayoría (58.2\%) mujeres, solteras procedentes de Estados Unidos, cuyo rango de edad estaba entre los 18-25 años, con un grado de escolaridad universitario, cuyo tiempo de permanencia fue de 1 a 2 meses con un gasto promedio de $\$ 1.500$ durante su estadía. El alojamiento que utilizaron fue en casas de familia de acogida y prefirieron los meses de diciembre a marzo por el factor climático de sus ciudades de origen y de mayo a julio por sus periodos de descanso; por otra parte, la mayoría de turistas idiomáticos escogieron estudiar en Cuenca por considerarla una ciudad accesible económicamente, culta y de buenos paisajes; además consideraron que la práctica del idioma español era muy buena por temas de pronunciación.

A continuación, en la tabla 1 se detalla, en breves rasgos, los resultados obtenidos en la investigación.

Tabla 1. Caracterización de la demanda

\section{Turistas idiomáticos}

Género

Nacionalidad

Edad

Estado civil

Escolaridad

Ocupación

Idioma

Otro idioma

Tiempo

de permanencia

Gasto promedio

Motivaciones

para estudiar español

Alojamiento

Por qué prefirió

Cuenca en esta época
La mayor parte de estudiantes idiomáticos son mujeres teniendo un porcentaje de $58.2 \%$.

El 74.2\% mayoritariamente son americanos.

La edad promedio varía entre los 18-25 años, teniendo un porcentaje de $72.58 \%$.

El $85.6 \%$ son solteros/as.

E1 77.9\% de extranjeros idiomáticos tienen estudios universitarios.

$73.5 \%$ son estudiantes.

La mayoría hablan inglés con un porcentaje de $78.6 \%$.

A más de su idioma natal, hablan bastante inglés $(4,64 / 5)$. En segundo lugar el idioma cuya calificación es regular es el italiano $(3,6 / 5)$.

El 53.2\% permanece en la ciudad por el lapso de 1-2 meses.

El gasto promedio tiene un rango de \$1-\$1.500 dólares según el $60.20 \%$ de encuestas respondidas.

El idioma y la cultura los motivó bastante para estudiar español, obteniendo una valoración de $(4,42 / 5)$, y $(4,21 / 5)$ respectivamente según la escala de Likert.

E1 74.9\% prefiere hospedarse en una casa de familia.

El $35.8 \%$ dice que prefirió venir porque el clima es agradable y el $30.1 \%$ porque tienen vacaciones. 
Cómo se enteró del centro de español

Recomendaría a la ciudad como destino idiomático

Por qué prefirió Cuenca sobre otros destinos idiomáticos

Con quién realizó su viaje

Otros tipos de turismo realizados

Regiones visitadas

Cómo obtuvo información para visitar Ecuador

El tiempo fue adecuado para aprender español
Los estudiantes dicen que la recomendación de amigos fue regular obteniendo una calificación de (3.11/5) en la escala de Likert.

E1 99\% de estudiantes dicen que si recomendarían a Cuenca-Ecuador como un destino de turismo idiomático.

El 22\% dice que Cuenca es bonita y barata, además el 16\% dice que el español de la ciudad es muy bueno.

El 46.8\% realizó el viaje para aprender español con sus amigos.

E1 33.3\% realizó actividades relacionadas al turismo cultural-histórico.

El 49.1\% de estudiantes encuestados dice que ha visitado la Sierra.

Los extranjeros idiomáticos dicen que la información obtenida de amigos fue regular $(3,5 / 5)$ en la escala de Likert.

El 72.58\% afirma que sí fue adecuado.

La recomendación más representativa con un porcentaje de $35 \%$ dice que todo está

Recomendaciones bien organizado y que los profesores son buenos, pero deben hablar más español en las clases.

\section{Discusión}

El perfil de los visitantes que realizan turismo idiomático en la ciudad de Cuenca en el año 2015 se define de la siguiente forma: mujeres, estadounidenses, solteras, universitarias, con una edad promedio de 18-25 años que vinieron por un periodo de tiempo de 1-2 meses y gastaron aproximadamente $\$ 1.500$ dólares, viajaron acompañadas de sus amigos y realizaron actividades culturales-históricas. El motivo que hizo que los visitantes idiomáticos se decidan por venir a la ciudad fueron puntualmente tres: el idioma, la cultura y la naturaleza, aunque el clima también fue un motivo de importancia. Prefirieron venir a Cuenca sobre otros destinos idiomáticos debido a que consideraron que el español es bastante bueno, la mayoría decidió alojarse en una casa de familia, lo que hizo que su inmersión lingüística sea un éxito total, por el constante intercambio cultural y lingüístico. También se concluyó que el turismo idiomático fue estacional, debido a que los meses más fuertes o en donde más afluencia de turistas idiomáticos hubo, fue el período comprendido entre diciembre-marzo y mayo-julio, esto no dependió de la oferta, sino más bien de la disponibilidad de tiempo de la demanda.

Durante el estudio se pudo notar que el medio por el cual se informaron del destino, fue básicamente por recomendaciones de amigos, más no por otros medios, es decir, si Cuenca tuvo un crecimiento y fortalecimiento en turismo idiomático fue gracias a los estudiantes que hicieron buenas recomendacio- nes más no por la promoción que se le daba a la ciudad en el extranjero. Los convenios que tenían los centros de enseñanza con universidades o centros de estudio extranjeros, también tuvieron mucho que ver con el crecimiento y explotación del turismo idiomático. Es preciso mencionar, también, que los centros de enseñanza, eran organizados y tenían buenos profesores, pero se debía mejorar, debido a que los estudiantes tenían el objetivo de aprender correctamente el español.

\section{Conclusiones}

De acuerdo a los datos recabados luego del estudio se concluyó que el turismo idiomático en la ciudad de Cuenca-Ecuador era un turismo con demanda creciente y por el cual se podía apostar para generar mayor ingreso económico en las personas que estaban vinculadas a la actividad, como las mismas escuelas de español, las casas de familia que los acogían y otros servicios turísticos que se veían beneficiados, además de generar también nuevos emprendimiento que involucren a las comunidades, en su mayoría comunidades campesinas cercanas a la ciudad, para aprovechar su rica cultura y tradición, generando a través de actividades ancestrales una oferta que complemente el estudio del español. .

El turismo idiomático genera un proceso de intercambio cultural el cual debe ser explotado $y$, al ser Cuenca-Ecuador una ciudad Patrimonio Cultural de la Humanidad, se debía aprovechar 
para dinamizar más la promoción turística del turismo idiomático, ya que la cultura era una de las motivaciones principales que hacía que los turistas escojan a la ciudad para lograr su objetivo de hacer la inmersión lingüística en el español, impulsando también todos los demás atractivos culturales de la ciudad, focalizado dicha promoción en los grupos de interés de acuerdo al perfil que se definió.

\section{Referencias}

Adriani, H. L., Leighton, G., Regina, S., Navarro, F., Jimeno, R., \& Arcuri, S. (2014). Una aproximación del turismo idiomático en Argentina. Reencuentro de saberes territoriales latinoamericanos.

Alonso, J. (2006). Naturaleza económica de la lengua. Instituto Complutense de Estudios Internacionales. Documento de trabajo.

Baraló, M. (2007). Enseñanza del español y turismo: las estancias lingüísticas. Mosaico. Revista para la promoción y apoyo a la enseñanza del español, 20, 32-36.

Baraló, M. (2015). El valor del idioma como recurso turístico, económico y cultural. Signos Universitarios, 34(51), 35-61.

Cohen, E., \& Cooper, R. L. (1986). Language and Tourism. Annals of Tourism Research, 13(4), 533-563.

Ganfornina, N. (2005). El turilingüismo en España: actitudes y preferencias de los estudiantes universitarios estadounidenses. Universidad Antonio de Nebrija.

Goeldner, C., \& Ritchie, B. (2011). Tourism: Principles, Practices, Philosophies. John Wiley \& Sons Inc.

Leighton, G., Adriani, H., \& Schüler, R. (2011). El turismo idiomático como industria cultural. $R e$ vista Geográfica de América Central, 2, 1-12.
También se concluye que los actores vinculados con esta actividad deberían trabajar inter-institucionalmente para crear planes y proyectos que sean de utilidad para mejorar la oferta y consolidar poco a poco la demanda creciente, para que tanto oferta y demanda se vean beneficiados por la actividad, dirigiendo esfuerzos en el grupo de interés que hemos definido a lo largo de la investigación.

Leighton, G., Arcuri, S., \& Jimeno, R. (2012). El turismo idiomático en la Argentina. Principales rasgos de la demanda. V Congreso Latinoamericano de Investigación Turística. San Pablo: Universidad de San Pablo.

Meza, L. (2013). Análisis del club del producto de turismo idiomático en la ciudad de Málaga. Universidad de Málaga.

Montaner, J. (1991). Estructura del mercado turístico. Madrid: Síntesis.

Moral Cuadra, S., \& Orgaz, F. (2013). Las nuevas tipologías del turismo en España: el caso de Andalucía. Eumed.

Pardo, C. (2011). El turismo idiomático en España como potenciador cultural en ciudades patrimoniales: El caso de Salamanca y Alcalá de Henares. Cuadernos de Turismo, 27, 701-723.

Pawlowska, E. (2011). El turismo académico. Un análisis económico para el caso de Galicia. Galicia. Santiago de Compostela: Universidade Servizo de Publicacións e Intercambio Científica.

Secretaría de Turismo. (2015). Secretaría de Turismo. Consultado 10 de diciembre, 2018. Obtenido de: www.sectur.gob.mx/hashtag/2015/05/14/ turismo-cultural/

Soto, F. (2012). La movilidad estudiantil internacional como turismo académico. Revista de Geografía de Valparaíso, 46, 54-68. 